\title{
CVA under Bates Model with Stochastic Default Intensity
}

\author{
Yaqin Feng \\ Department of Mathematics, Ohio University, Athens, USA \\ Email: fengy@ohio.edu
}

How to cite this paper: Feng, Y.Q. (2017) CVA under Bates Model with Stochastic Default Intensity. Journal of Mathematical Finance, 7, 682-698.

https://doi.org/10.4236/jmf.2017.73036

Received: June 26, 2017

Accepted: July 28, 2017

Published: July 31, 2017

Copyright (C) 2017 by author and Scientific Research Publishing Inc. This work is licensed under the Creative Commons Attribution International License (CC BY 4.0).

http://creativecommons.org/licenses/by/4.0/

\section{cc) (i) Open Access}

\begin{abstract}
Counterparty credit risk has received increasing attention and become a topical issue since 2007 credit crisis, particularly for its impact on the valuation of the OTC derivatives. Credit Value Adjustment (CVA) has become an import field and it is required in Basel III. This paper studies CVA for European options under Bates model with stochastic default intensity. We develop a Monte Carlo and finite difference method framework for assessing exposure profiles and impact of counterparty credit risk in pricing. The exposures are computed by solving a partial integro-differential Equation (PIDE) using implicit-explicit (IMEX) time discretization schemes. CVA in presence of wrong way risk (WWR) is embedded in the correlation between risk factor and default intensity. Meanwhile, the jump-at-default feature of the models offers an effective means to assess WWR. Our results show that both jump and WWR play an important role in evaluating CVA and exposures. The impact is significant and it is crucial for risk management purpose.
\end{abstract}

\section{Keywords}

CVA, Bates Model, Stochastic Default Intensity, Wrong Way Risk, Jump at Default

\section{Introduction}

Counterparty credit risk (CCR) refers to the risk that a counterparty of a financial contract will default prior to the expiration of the contract, and thus cannot make the required contractual payments. It has been widely considered as one of the key drivers of the 2007-2008 financial crisis. The management of counterparty credit risk has caught special attention since the financial crisis. As pointed out by Basel Committee on Banking Superversion [1] [2]: "During the financial crisis, banks suffered significant counterparty credit risk (CCR) losses on their OTC derivatives portfolios. The majority of these losses came from fair value 
adjustment on derivatives". Regulations presented in the Basel III include credit valuation adjustment (CVA) as an additional capital charge requirement. CVA is defined as the difference between the risk free value of a contract and the market value of the contract with the possibility of a defaulting counterparty [3].

One difficulty in pricing CVA arises from the uncertainty of the losses given the default, which is known as exposure. The exposure of contracts evolves before the expiration, diverting away from the initial value. Because of the growing practical importance, there are recently articles that discuss the computation of the exposure. Feng and Oosterlee (2012) [4] studied the exposure profile of European, Bermudan and barrier options under the Heston and Heston HullWhite model. Graaf et al. (2014) [5] presented various techniques to approximate exposure for early exercise option within Black-Scholes and Heston framework. Graaf et al. (2014) [6] explored the exposure profiles and CVA sensitivities for Barrier option in the context of Black-Scholes and Heston model.

The other difficulty in pricing CVA concerns the dependency between exposure and counterparty credit quality, which is known as wrong/right way risk (WWR). In the context of computation of CVA, the correct inclusion of Wrong Way Risk (WWR) is still a major concern. Many different approaches have been proposed to assess WWR. Hull and White (2012) [7] adjusted default probability in the dependent CVA formula. Pykhtin and Rosen (2010) [8] used copula method to model the dependence between default time and exposures. Alternative method such as change of measure is illustrated in Brigo and Vrins (2016) [9]. We focus on the dependence between the counterparty default and general market risk factors.

Since stochastic volatility models such as Heston model and Bates model are widely used in practice, it is often of interest to estimate CVA under the stochastic volatility model and analyze the impact of stochastic volatility on CVA. Comparing to Heston model, Bates extended Heston model by adding a jump term in the stock process. The inclusion of jumps is necessary for stochastic volatility models to comply with the market observed phenomena to varying degrees. At the same time, jumps modeling the sudden changes in the risk factors and it could be related to counterparty's default. Some of market factors will jump immediately following the default event [10]. Bates model in the presence of jump-to-default is the joint modeling of equity and credit model. The jump model conditional on default for CVA was discussed in [10]. Fabio and Minqiang (2015) [11] investigated CVA in the presence of WWR by introducing jumps at default to model. Unlike other models, we embed the relation between default and risk factors in the model by the jump.

In this paper we model CVA for European options under Bates model with stochastic default intensity which presents several attractive features.

Firstly, Bates model is a stochastic jump diffusion model. Jump diffusion are capable of modeling large and sudden changes in the state variable [12]. Default and risk factors are correlated through jump. It is intuitive and realistic [11]. Secondly, under the Monte Carlo framework, by modeling a stochastic intensity 
model, default and risk factors can be easily connected through correlations from Brownian motion. Default and risk factors are then further correlated through correlation parameter. It is very easy to implement. Thirdly, option values in the model are computed by finite difference method of the corresponding PIDE. Both Heston model and jump diffusion models are actually nested by Bates model. It is flexible.

We develop a PDE based Monte Carol framework for pricing CVA and assess exposure profile. The framework contains essentially three steps: risk factor simulation, independent exposure estimation and WWR incorporation. Monte Carlo method is used to generate asset paths from initial time to maturity. Along the paths, independent option values are determined at each time grid by PDE method. WWR is then formulated by introducing a kind of model-to market survival rate change ratio. Under this framework, CVA for both single trade and portfolios can be treated.

This paper contributes as follows: first we expand Bates model by modeling the intensity of jump to default with the stochastic process, i.e., the CIR++ process which provides a natural and effective framework to handle the correlation between the underlying asset and the default, evaluate the impact on exposure and CVA and assess WWR; second we develop an efficient PDE based Monte Carlo framework for pricing CVA and assessing exposure profile under Bates model with stochastic intensity of the jump to default which combines advantages of Monte Carlo simulation (such as path-wise pricing, and properly netting and collateral modeling) and efficiency of PDE pricing. Our framework can be used for both single trade and portfolios; with the developed efficient framework, our results show that both jump and WWR play an important role in evaluating CVA and exposures. The impact is significant and it is crucial for risk management purpose.

The outline of the paper is as follows. In Section 2, we describe CVA valuation problem in general terms. Section 3 describes the stochastic intensity model and underlying asset driven process. In Section 4, we present the general framework for CVA pricing based on Monte Carlo and PDE method. In Section 5, we present test results. Conclusion is summarized in Section 6.

\section{Preliminary}

\subsection{CVA, EE and PFE}

Counterparty risk is very similar to other forms of credit risk in that the economic loss is obligor's default. There are two features that set counterparty risk different from more traditional forms of credit risk: the uncertainty of exposure and the bilateral nature of credit risk [13]. In the following, we will review the definition of credit exposure and CVA.

In the event that a counterparty has defaulted, an institution may close out the relevant contracts and cease any future payments. Following this, they may determine the net amount owing between them and their counterparty. If the net amount is negative, the institution is in debt to its counterparty and is still legally 
obliged to settle this amount. Hence, from a valuation's perspective, the position appears essentially unchanged. The institution does not gain or loss from the counterparty's default. If the net amount is positive, the institution will have a claim on the positive value at the time of default [3]. Thus the credit exposure of an institution that has a trade level contract with a counterparty is given by

$$
E(t)=\max \left\{U_{t}, 0\right\},
$$

where $U_{t}$ is the portfolio's market value at $t$. Uncertain future exposure can be visualized by means of exposure profiles. This leads to the definition of expected exposure. Assume that all the stochastic processes considered are defined on the probability space $\left(\Omega, \mathcal{F}_{t}, Q\right)$, where $Q$ is the risk neutral measure and $\mathcal{F}_{t}$ is the filtration up to time $t$. Expected exposure (EE) at a future time is given by:

$$
E E(t)=E\left\{U_{\mathrm{t}}^{+} \mid \mathcal{F}_{0}\right\},
$$

where $U_{t}^{+}=\max \left(U_{t}, 0\right)$ and $E$ denotes the expectation under the risk-neutral measure $Q$.

Potential future exposure (PFE) is the maximum loss due to counterparty default with a given confidence level. It is the quantile of the exposure at a certain level and is used to measure the "worst" loss for the risk management purpose. The mathematical definition is given by:

$$
\operatorname{PFE}(t)=\inf \left\{x \mid Q\left(U_{t}<x \mid \mathcal{F}_{0}\right)>\alpha\right\}
$$

where $\alpha$ is the confidence level. For calculating PFE, $\alpha=97.5 \%$ is commonly used to measure the worst losses.

Credit value adjustment (CVA) is by definition the difference between risk free portfolio value and the true portfolio value that takes into account the possibility of counterparty's default [13]. If the counterparty defaults, the institution will be able to recover a constant fraction of exposure which is denoted by $R$. Denote the time of counterparty default by $\tau$, then

$$
C V A=(1-R) E\left[P(0, \tau) \cdot U_{\tau}^{+} \cdot 1_{\{\tau \leq T\}}\right]
$$

where $P(0, \tau)$ denotes the discount factor for maturity $\tau$ and $T$ is maturity. Assume survival probability

$$
G(t):=Q(\tau>t)=E\left[1_{\{\tau>t\}}\right]
$$

then the expression for CVA becomes

$$
C V A=-(1-R) \int_{0}^{T} P(0, t) \cdot E\left[U_{t}^{+} \mid \tau=t\right] \mathrm{d} G(t)
$$

This is the general CVA formula. As we can see, in Equation (6), one key element to calculate CVA is the conditional expectation $E\left[U_{t}^{+} \mid \tau=t\right]$. Therefore, the dependence between $U_{t}$ and default time $\tau$ is important and can be material. In general, the dependency between exposure and counterparty credit quality is known as wrong/right way risk (WWR).

Assume exposure and counterparty default are independent, Equation (6) can be simplified to: 


$$
C V A=-(1-R) \int_{0}^{T} P(0, t) \cdot E\left[U_{t}^{+}\right] \mathrm{d} G(t) .
$$

This is the independent CVA formula.

In this paper, we will not only focus on the calculation of exposure, but also investigate the impact of WWR on CVA.

\subsection{CVA, EE with WWR}

According to the International Swaps and Derivatives Association, WWR is defined as the risk that occurs when the exposure to a counterparty is adversely correlated with the credit quality of that counterparty [14]. Many different techniques have been proposed to assess WWR. Brigo and Pallavicini (2008) [15] studied a stochastic intensity and its correction with risk factors. Pykhtin et al. (2010) [8] and Bocker et al. (2014) [14] use copula to model the WWR. Hull and White (2012) [7] proposed a deterministic relationship that links higher values of exposure paths with a higher default probability. Bocker et al. (2014) [14] and Ruiz et al. (2015) [16]) modeled WWR by introducing different risk weights for different exposure scenarios. Brigo and Vrins (2016) [9] introduced wrong way measure and tackled WWR through change of numeraire.

As we know, survival probability $G(t)$ defined in Equation (5) is a deceasing function satisfying $G(0)=1$. Survival probability $G(t)$ can be expressed in terms of hazard rate $h(t)$ and the relation between $G(t)$ and $h(t)$ is $G(t)=\mathrm{e}^{-\int_{0}^{t} h(u) \mathrm{d} u}$. Following the stochastic intensity model in [9], we introduce a survival process $M_{t}=Q\left(\tau>t \mid \mathcal{F}_{t}\right)$ on the probability space $\left(\Omega, \mathcal{F}_{t}, Q\right)$, and assume that:

$$
M_{t}=\mathrm{e}^{-\int_{0}^{t} \lambda_{u} \mathrm{~d} u},
$$

where $\lambda_{t}$ is a stochastic process. Formally, the survival process $M_{t}$ and survival probability $G(t)$ are linked by

$$
E\left(M_{t}\right)=G(t) .
$$

As mentioned in [9], CVA with WWR in this setting reduces to:

$$
C V A=-(1-R) \int_{0}^{T} P(0, t) \cdot E\left[U_{t}^{+} \zeta_{t}\right] \mathrm{d} G(t)
$$

where

$$
\zeta_{t}:=\frac{\lambda_{t} M_{t}}{E\left(\lambda_{t} M_{t}\right)}
$$

The denominator $E\left(\lambda_{t} M_{t}\right)$ can be considered as the prevailing market view of default likelihood and $E\left(\zeta_{t}\right)=1$. The process $\zeta_{t}$ is a kind of model-tomarket survival rate change ratio [9]. For more discussion of $\zeta_{t}$, we refer to [9]. In the above expression, expected exposure $E\left[U_{t}^{+}\right]$with consideration of WWR will be $E\left[U_{t}^{+} \zeta_{t}\right]$. It is a weighted exposure and the exposure weight function is determined by $\zeta_{t}$. Note that this definition is consistent with the WWR discussion in [14]. Unless otherwise specified, Equation (9) is used to estimate CVA and EE for the paper. 


\section{Modeling Assumptions}

In this paper, in order to quantify WWR for CVA, we consider a stochastic intensity model, namely Cox-Ingersoll-Ross (CIR) model for the counterparty. The underlying process is driven by Bates models. WWR is modeled by a joint simulation of the underlying processes that drive counterparty exposure and credit risk. The dependence structure is modeled by the correlation between driven Brownian motion as well as the jump-to-default feature of Bates model. We assume deterministic interest rate $r$ and hence deterministic discount factor $P(0, \tau)$, but all our conclusions hold under stochastic interest rates that are independent of default time.

\subsection{Cox-Ingersoll-Ross (CIR)}

In this paper, we assume $\lambda_{t}$ follows CIR process. The hazard rate process $\lambda_{t}$ can be formulated as follows:

$$
\begin{aligned}
& \lambda_{t}=y_{t}+\psi_{t} \\
& \mathrm{~d} y_{t}=\kappa_{c i r}\left(\theta_{c i r}-y_{t}\right) \mathrm{d} t+\sigma_{\text {cir }} \sqrt{y_{t}} \mathrm{~d} B_{t},
\end{aligned}
$$

where $y_{t}$ follows a standard CIR process and the deterministic function $\psi_{t}$ allows calibration to the current hazard curve. The parameters $\kappa_{c i r}, \theta_{c i r}, \sigma_{c i r}$ and $y_{0}$ are positive deterministic constant. As usual, $B_{t}$ is standard Brownian motion under the risk neutral measure. It is well known that the probability density of $y_{t}$ is given by a non-central chi-square distribution. The closed form density leads to a closed form formula for the survival probability formula. Let $P(t, T ; y)$ be the survival probability up to time $T$ conditional on survival up to time $t$, then

$$
P(t, T ; y)=E\left[\mathrm{e}^{-\int_{t}^{T} y_{s} \mathrm{~d} s}\right]=A(t, T) \mathrm{e}^{-B(t, T) y_{t}},
$$

where

$$
\begin{gathered}
A(t, T)=\left(\frac{2 h e^{\left(\kappa_{c i r}+h\right)(T-t) / 2}}{2 h+\left(\kappa_{c i r}+h\right)\left(\mathrm{e}^{(T-t) h}-1\right)}\right)^{2 \kappa_{\text {cir }} \theta_{\text {cir }} / \sigma_{\text {cir }}^{2}}, \\
B(t, T)=\frac{2\left(\mathrm{e}^{(T-t) h}-1\right)}{2 h+\left(\kappa_{\text {cir }}+h\right)\left(\mathrm{e}^{(T-t) h}-1\right)},
\end{gathered}
$$

and $h=\sqrt{\kappa_{\text {cir }}^{2}+2 \sigma_{\text {cir }}^{2}}$.

\subsection{CVA under Bates Model}

We will present methods for computation of the exposure of European options under the Bates model [17]. Bates model combines the Merton jump model [18] and the Hestonstochastic volatility model [19]. Heston model and jump diffusion models are actually nested by Bates model. Bates model describes the behavior of the asset value $S_{t}$ and its variance $V_{t}$ by the following SDE: 


$$
\begin{aligned}
& \mathrm{d} S_{t}=\left(r-\lambda_{J} \zeta\right) S_{t} \mathrm{~d} t+\sqrt{V_{t}} S_{t} \mathrm{~d} W_{t}^{1}+(J-1) S_{t} \mathrm{~d} N(t) \\
& \mathrm{d} V_{t}=\kappa\left(\theta-V_{t}\right) \mathrm{d} t+\sigma \sqrt{V_{t}} \mathrm{~d} W_{t}^{2} \\
& \left\langle\mathrm{~d} W_{t}^{1}, \mathrm{~d} W_{t}^{2}\right\rangle=\rho_{m} \mathrm{~d} t
\end{aligned}
$$

where $r$ is the risk free interest rate, $N(t)$ is the standard Poisson process which models numbers of jumps and has intensity $\lambda_{J}$. Jump size of the Poisson process is denoted by $J . J$ follows log-normal distribution with parameter and its probability density function is denoted by $f(J)$. The relation between $\zeta$ and $J$ is: $\zeta=\mathrm{e}^{\mu_{J}+\frac{1}{2} \sigma_{J}^{2}}-1 . \kappa$ is the rate of mean reversion level, $\theta$ is the mean level of variance and $\sigma$ is the volatility of $V(t) . W_{t}^{1}$ and $W_{t}^{2}$ are standard Brownian motion processes with correlation $\rho_{m}$.

Price $U$ of an option with maturity $T$, payoff function $g\left(S_{T}, V_{T}\right)$ and with the initial value of the underlying and volatility to $S$ and $V$ respectively equals: $U(S, V, t)=E\left[\mathrm{e}^{-r(T-t)} g\left(S_{T}, V_{T}\right) \mid S_{0}=S, V_{0}=V\right]$. Since it's a two dimensional pricing problem and its analytical formula is hard to obtain, we will apply finite difference method to solve the associate PDE under the CVA framework. Therefore, from Equation (2), expected exposure in Beta's model can be expressed as

$$
E E(t)=E\left\{\max \left(U\left(S_{t}, V_{t}, t\right), 0\right) \mid \mathcal{F}_{0}\right\}
$$

\subsection{WWR CVA}

We now move to the computation of the CVA, as in Equation (9).

In the case study below, we assume the correlation between $B_{t}$ and $W_{t}^{1}$ is $\rho$, i.e. $\left\langle\mathrm{d} B_{t}, \mathrm{~d} W_{t}^{1}\right\rangle=\rho \mathrm{d} t$. Note that $\rho$ and $\rho_{m}$ are different. $\rho_{m}$ is the correlation between the asset value $S_{t}$ and its variance $V_{t}$, while $\rho$ reflects the correction between the underlying process and the default intensity. Furthermore, we assume $\left\langle\mathrm{d} B_{t}, \mathrm{~d} W_{t}^{2}\right\rangle=0$. It is clear that when $\rho=0$, $E\left[U_{t}^{+} \zeta_{t}\right]=E\left[U_{t}^{+}\right] E\left[\zeta_{t}\right]=E\left[U_{t}^{+}\right]$. Here the second equality comes from the fact that $E\left(\zeta_{t}\right)=1$. CVA expression in Equation (9) reduces to Equation (7), which is the independent CVA formula. The impact of the WWR manifests itself in the term $U_{t}^{+} \zeta_{t}$ from the correlation parameter $\rho$.

As we know, Bates model in the presence of jump-to-default is the joint modeling of equity and credit model. WWR is also modeled by introducing jumping at default part. To measure WWR from the jump contribution, we simply remove the jump part from Bates model, which is actually Heston model.

\section{General Scheme for CVA Pricing for under Bates Model with Stochastic Intensity}

We assume constant recovery rate $R$ and independence between interest rate and other factors. CVA formula in Equation (9) then has following key components: the estimation of exposure $V_{t}$, the calculation of survival ratio $\zeta_{t}$ and the final estimation of CVA. For CVA calculation purpose, we are not only concerned with option value at time $t=0$, but also for all the time till expiration of 
the contract. When PDE method is used to price a single trade, only a single grid point in initial time is used. In order to calculate exposure at all future time, the finite difference method uses a large portion of the grid points. It makes the PDE method computationally attractive [6]. Therefore, we will employ PDE method to find exposure. The estimation of $\zeta_{t}$ is accomplished through MC simulation. In the following, we build MC based PDE framework to price CVA and discuss practical implementation of the jump-to-default feature CVA.

\subsection{The Finite Difference Method for Exposure Calculation}

For a European option with maturity $T$ and payoff function $g\left(S_{T}, V_{T}\right)$, the risk-neutral price at any time $t \leq T$ is:

$$
U(S, V, t)=E\left[\mathrm{e}^{-r(T-t)} g\left(S_{T}, V_{T}\right) \mid S_{t}=S, V_{t}=V\right] .
$$

Let $\tau=T-t$, then $U(S, V, \tau)$ satisfies the following partial integro-differrential Equation (PIDE):

$$
\begin{aligned}
\frac{\partial U(S, V, \tau)}{\partial \tau}= & \frac{1}{2} V S^{2} \frac{\partial^{2} U(S, V, \tau)}{\partial S^{2}}+\rho_{m} \sigma V S \frac{\partial^{2} U(S, V, \tau)}{\partial S \partial V} \\
& +\frac{1}{2} \sigma^{2} V \frac{\partial^{2} U(S, V, \tau)}{\partial V^{2}}+\left(r-q-\lambda_{J} \zeta\right) S \frac{\partial U(S, V, \tau)}{\partial S} \\
& +\kappa(\theta-V) \frac{\partial U(S, V, \tau)}{\partial V}-\left(r+\lambda_{J}\right) U(S, V, \tau) \\
& +\lambda_{J} \int_{0}^{\infty} U(J S, V, \tau) f(J) \mathrm{d} J
\end{aligned}
$$

where $f(J)$ is log-normal probability density function. By introducing operators

$$
\begin{aligned}
L_{c} U= & \frac{1}{2} V S^{2} \frac{\partial^{2} U}{\partial S^{2}}+\rho_{m} \sigma V S \frac{\partial^{2} U}{\partial S \partial V}+\frac{1}{2} \sigma^{2} V \frac{\partial^{2} U}{\partial V^{2}} \\
& +\left(r-q-\lambda_{J} \zeta\right) S \frac{\partial U}{\partial S}+\kappa(\theta-V) \frac{\partial U}{\partial V}-\left(r+\lambda_{J}\right) U
\end{aligned}
$$

and

$$
L_{J} U=\lambda_{J} \int_{0}^{\infty} U(J S, V, \tau) f(J) \mathrm{d} J
$$

the partial integro-differential equation in (17) can be written as :

$$
\frac{\partial U}{\partial \tau}=L_{c} U+L_{J} U
$$

The RHS of Equation (20) can be split into two parts. The first part is a differential operator $L_{c}$, which can be discretized in a similar way to Heston PDE. The second part $L_{J}$ is an integral term and need to be evaluated.

\subsubsection{Time Discretization}

The numerical solution of Equation (20) is straightforward since applying a standard space discretization lead to a tridiagonal matrix. However, the presence of the jump terms which results in PIDE causes the discretization matrix to be full. Therefore, we adopt implicit-explicit (IMEX) time discretization schemes. 
The jump term is treated explicitly, while the rest is handled implicitly. Let us construct $N+1$ grid points in time direction with step size $\Delta \tau=\frac{T}{N}$. By applying IMEX scheme, we have

$$
\frac{U_{n+1}-U_{n}}{\Delta \tau}=L_{c} U_{n+1}+L_{J} U_{n}
$$

\subsubsection{Discretization of $L_{c}$}

The discretization for differential operator $L_{c}$ is similar to the discretization of Heston PDE operator. Solving Heston PDE to price European and American option is extensively studied in [20] [21]. We follow the scheme mentioned in [21] for discretization. We use a computational grid that is uniform in $\tau$ and nonuniform in $S$ and $V$. Central schemes have been used to estimate the first derivative, second derivative and mixed derivative. Upwind scheme is applied on the boundary point. This is a quite well known method and we do not show the detailed here. For more details, we refer to [21].

\subsubsection{Discretization of $L_{J}$}

The integral term in operator $L_{J}$ need to be estimated in each of the grid points $0=S_{\min }, S_{1}, S_{2}, \cdots, S_{n}=S_{\max }$. We follow the same procedure as listed in [22] [23]. Let

$$
I_{i}=\int_{0}^{\infty} U\left(J S_{i}, V, \tau\right) f(J) \mathrm{d} J
$$

by introducing the change of variable $J=\mathrm{e}^{x}$ and decomposing the integration interval into $\left[0, S_{\max }\right]$ and $\left[S_{\max }, \infty\right]$, Equation (22) becomes

$$
I_{i}=\sum_{k=1}^{n} I_{i, k}+\int_{\ln \left(\frac{S_{\max }}{S_{i}}\right)} \mathrm{e}^{x} U\left(\mathrm{e}^{x} S_{i}, V, \tau\right) p(x) \mathrm{d} x .
$$

where $p(x)$ is normal probability density function with mean $\mu_{J}$ and standard deviation $\sigma_{J}$ and

$$
\begin{aligned}
I_{i, k} & =\int_{\ln \left(\frac{S_{k}}{S_{i}}\right)}^{\ln \left(\frac{S_{k+1}}{S_{i}}\right)} U\left(\mathrm{e}^{\mathrm{x}} S_{i}, V, \tau\right) p(x) \mathrm{d} x \\
& \approx \frac{1}{2} \ln \left(\frac{S_{k+1}}{S_{k}}\right)\left[U\left(S_{k}, V, \tau\right) p\left(\ln \left(\frac{S_{k}}{S_{i}}\right)\right)+U\left(S_{k+1} V, \tau\right) p\left(\ln \left(\frac{S_{k+1}}{S_{i}}\right)\right)\right] .
\end{aligned}
$$

The interval $\left[S_{\min }, S_{\max }\right]$ is chosen to be large so that the last integral in Equation (23) is negligible.

\subsection{Monte Carlo Simulation for Stochastic Intensity}

For realistic implementation of hazard rate process in CVA context, we rely on Monte Carlo simulate to generate the path for hazard rate. Several schemes have been tested for simulating the CIR process. Most of them are comparable when Feller condition is satisfied and when volatility is small. However, when the volatility is large, the performances deteriorate [9]. One can use reflected schemes to avoid negative samples along the path, however, the time step required to en- 
sure the convergence is too small. Alternatively, the scheme mentioned in [24] seems to work well. It consists of the following scheme for discretization:

$$
\begin{aligned}
y_{i+1}= & \left(\left(1-\frac{\kappa_{c i r}}{2}\left(t_{i+1}-t_{i}\right)\right) \sqrt{y_{i}}+\frac{\sigma_{c i r}\left(W_{i+1}-W_{i}\right)}{2\left(1-\frac{\kappa_{c i r}}{2}\left(t_{i+1}-t_{i}\right)\right)}\right)^{2} \\
& +\left(\kappa_{c i r} \theta_{c i r}-\frac{\sigma_{c i r}^{2}}{4}\right)\left(t_{i+1}-t_{i}\right)
\end{aligned}
$$

The scheme above allows violating the Feller constraint somewhat without loss of probability at or through the boundary. We will use this scheme to simulate the path for hazard rate.

\subsection{CVA Calculation Based on Monte Carlo and PDE Method}

For the purpose of CVA calculation, we first use Monte Carlo simulation to generate market state variables. Next, a grid in $S$ and $V$ is created. Price at each grid is calculated through PDE method. For any time $t$ which is not in line with simulation time, option price is obtained by bilinear interpolation on the grids. At each time point, expected exposure can be calculated accordingly. The flow chart of the procedure is given in Figure 1. In a summary, the basic procedure is presented in following steps:

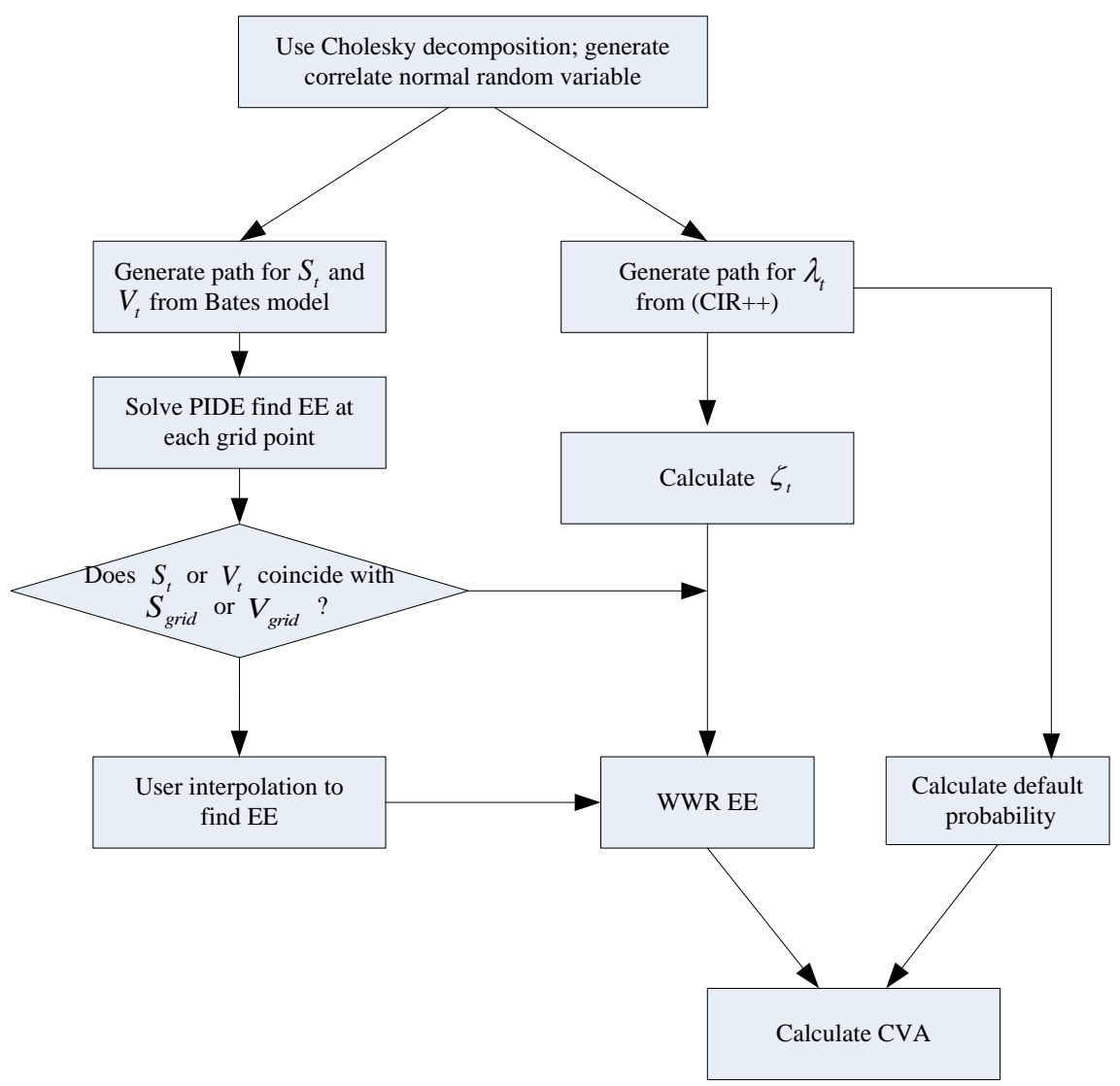

Figure 1. Flow chart. 
1) Generate path for $S_{t}$ and $V_{t}$ along time axis for $\operatorname{SDE}(14)$;

2) Generate path for hazard rate $\lambda_{t}$ using the Scheme (25) and estimate $\zeta_{t}$ use Equation (10);

3) Calculate option values at all PDE grids for all time $t$;

4) Calculate option values for all $S_{t}, V_{t}$ at all time $t$. If $S_{t}$ or $V_{t}$ doe not coincide with $S_{\text {grid }}$ or $V_{\text {grid }}$, bilinear interpolation will be applied;

5) Calculate EE, PFE from all the path at each time;

6) Calculate CVA.

\section{Numerical Results}

In this section, we price EE and CVA for European options under Bates model with stochastic default intensity. In order to study the impact of WWR, we show the EE and CVA for vanilla European option. Following this, we discuss the impact of the model parameters on CVA.

For all tests we covered, we follow the basic procedures listed in Section 4.3. In order to generate Monte Carlo paths for market risk factors, the jump and diffusion parts of the underlying asset under the Bates model can be simulated separately and multiplied together at the end. To simulate the diffusion part of the underlying asset, we will use Quadratic Exponential (QE) scheme.

Unless otherwise specified, the parameters for European call option are given by: $T=2, r=0, q=0, S_{0}=100$. The model parameters employed in numerical experiments are listed in Table 1. In addition, the number of Monte Carlo paths is set to be 10,000 , and the time step size of the SDE discretization $\Delta t=0.01$.

\subsection{WWR CVA from Parameter $\rho$}

To analyze the impact of the WWR on EE and CVA, we provide EE profile and CVA for different choices of $\rho$. To isolate other factor's impact, we only vary parameter $\rho$ and keep other parameters as listed in Table 1. Figure 2 contains the numerical results for the at the money(ATM) option with strike $=100$, while Figure 3 refers to the in the money(ITM) case with strike $=80$, and Figure 4 displays the result for out of the money $(\mathrm{OTM})$ European option with strike $=$ 120. Left panel of each figure denotes the EE profile for different levels of $\rho$, right panel is the corresponding CVA plot. From those figures, we can see the following:

When $\rho=0$, EE obtained by CVA with WWR and no WWR are extremely close and the differences are within Monte Carlo error. As we know, when the

Table 1. Base parameters.

Betas model

$$
\kappa=2, \theta=0.04, \sigma=0.25, \rho_{m}=0, V_{0}=0.04,
$$

$$
\lambda_{J}=0.2, \quad \mu_{J}=-0.5, \sigma_{J}=0.2
$$

CIR model

$$
\kappa_{\text {cir }}=0.34, \theta_{\text {cir }}=0.12, \quad \sigma_{\text {cir }}=0.13, y_{0}=0.15
$$


EE WWR

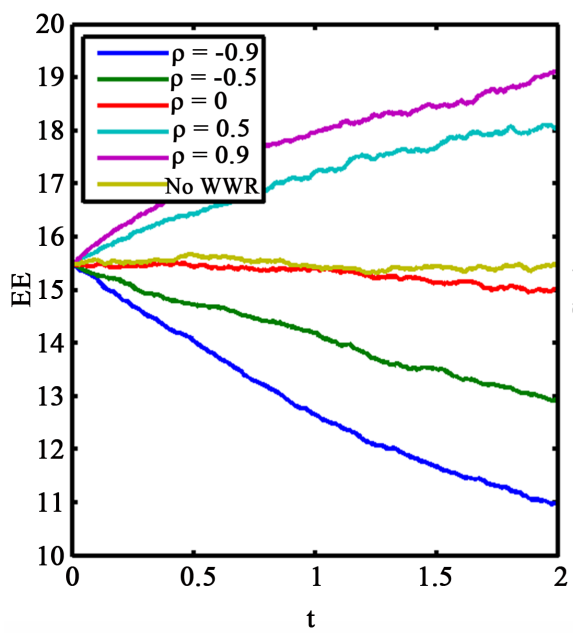

Figure 2. ATM.

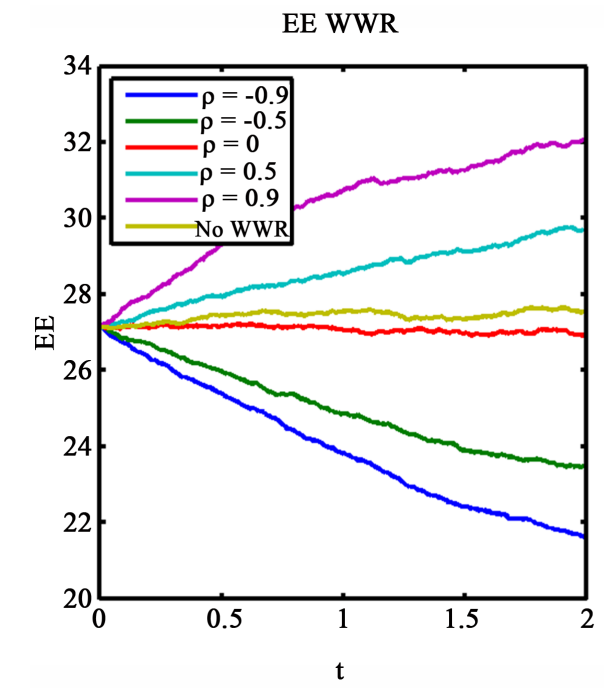

Figure 3. ITM.

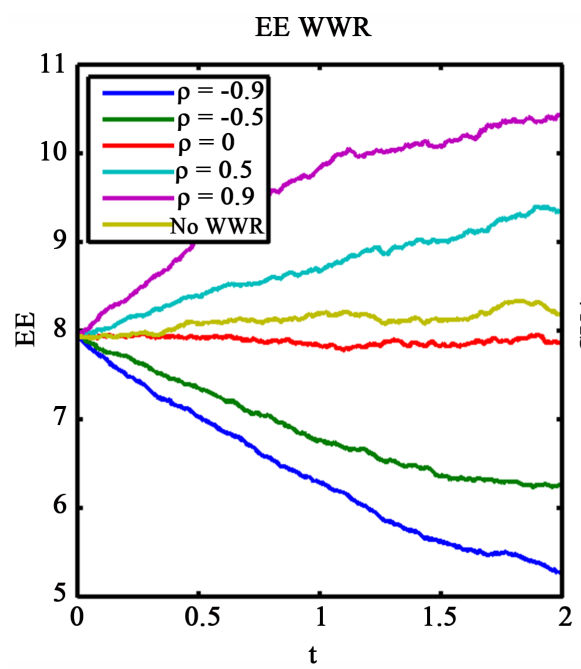

CVA

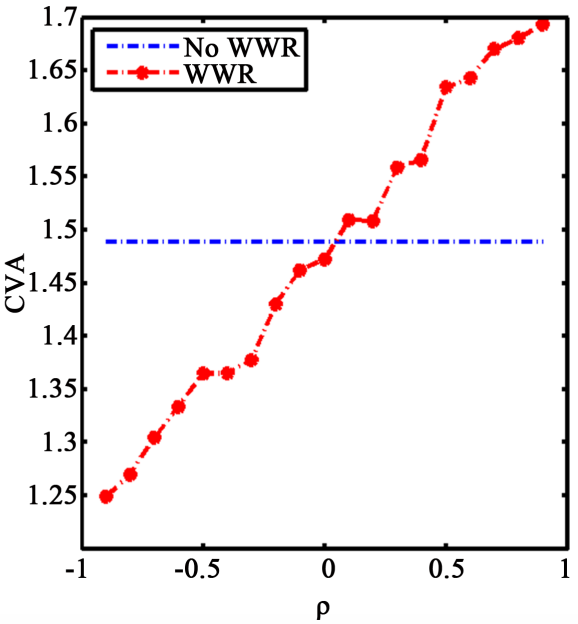

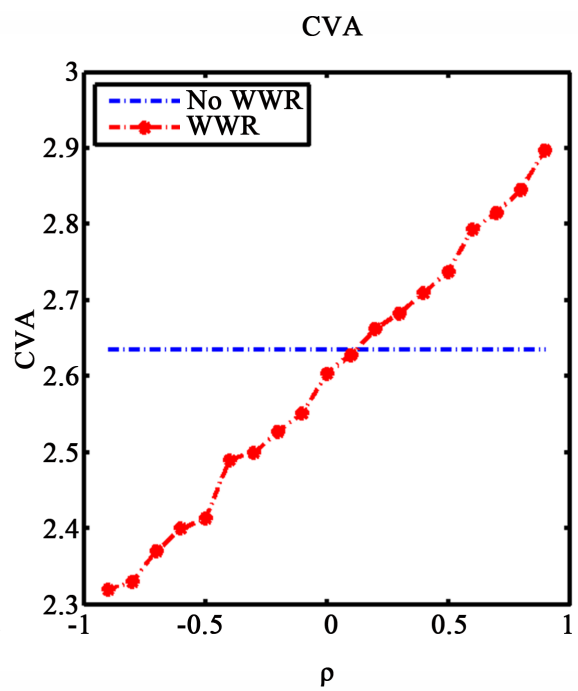

Figure 4. OTM.

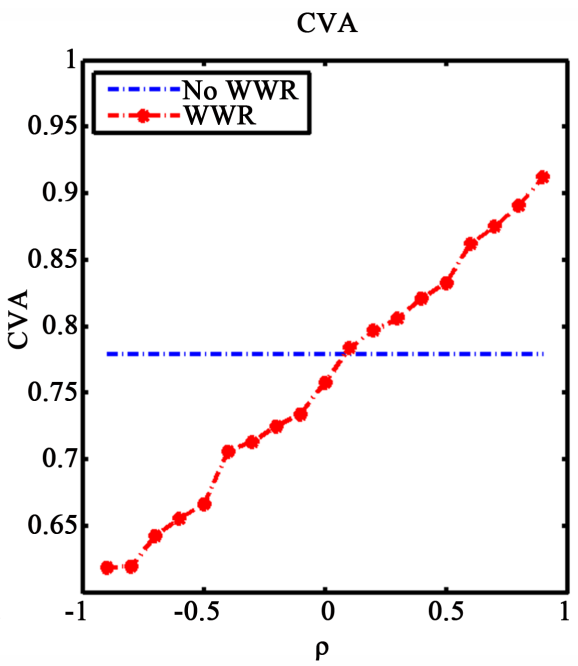


correction is zero, EE with WWR should be the same as EE with no WWR. Test results are as expected. Sizable differences are observed between EE and CVA with the consideration of WWR when $\rho \neq 0$. WWR CVA is $15.01 \%$ higher than the independent CVA for $\rho=0.9$ and $15.16 \%$ lower than it for $\rho=-0.9$ for ATM option. For OTM case, the difference is $20.36 \%$ and $18.37 \%$ for $\rho=0.9$ and $\rho=-0.9$. For ITM case, similar size of differences are observed as well. All the results show that the WWR introduced through correlation has an impact on CVA.

\subsection{WWR CVA from the Jump Effect}

In this part, we assess the impact of jump on EE and PFE profiles. We start with the comparison of Heston model to Bates model on EE and PFE profile. Then we conduct a detailed analysis of jump impact on CVA. Basically, we stress the jump parameters from Bates model by considering different levels of jump intensity and jump size volatility. Note that the parameters are chosen from Table 1.

\subsubsection{Effect of Jump}

In this part, we consider the overall jump impact to CVA. As we mentioned in Section 3.3, in order to quantify the WWR from jump contribution, we remove the jump part from Bates model (which is Heston model) and compare EE and CVA between these two models. Test results are illustrated in Figures 5-7. When the results for Heston model are compared to Bates model, significant difference can be noticed for both EE and PFE. The average EE difference are $29.0 \%, 24.7 \%$ and $28.1 \%$ for $\rho=0,-0.9$ and 0.9 respectively. For PFE, the difference grows as time elapses. All the differences are introduced by model difference (the jumps).

Let us now focus on the Figure 5 when $\rho=0$, this is an independent CVA case. Independent of underlying dynamics, EE starts at initial option value and oscillates around initial value and return to the initial level at expiry. It can be
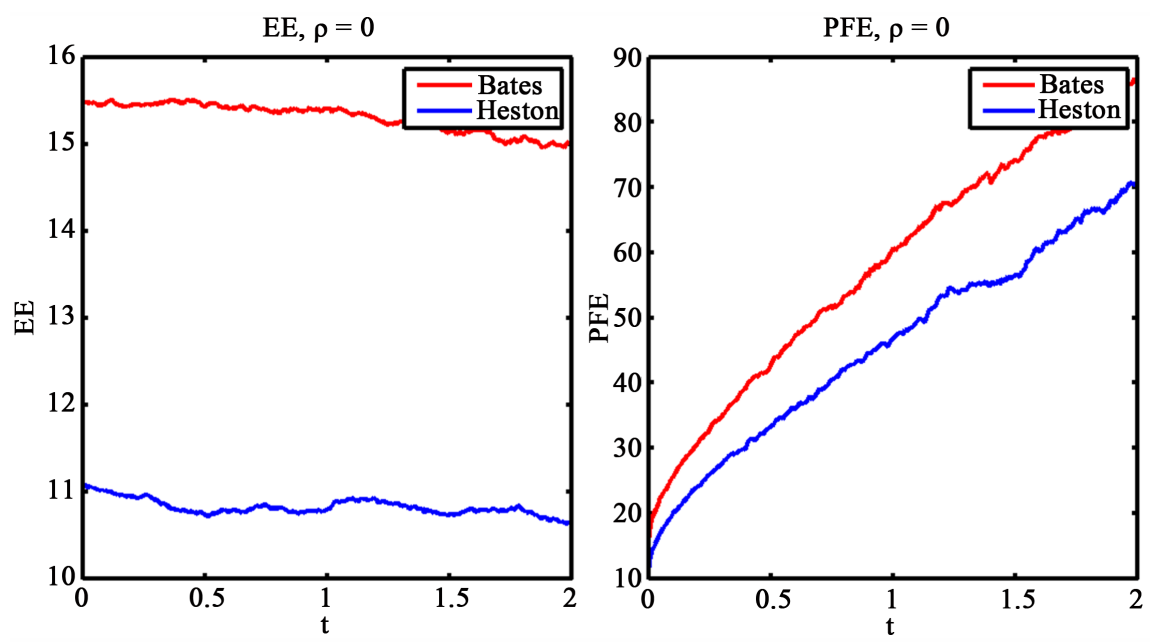

Figure 5. ATM option, $\rho=0$. 
$\mathrm{EE}, \rho=0.9$

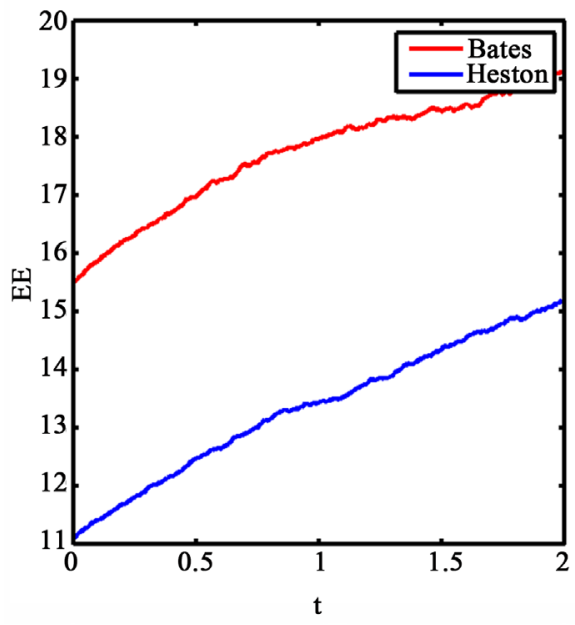

Figure 6. ATM option, $\rho=0.9$.
EE, $\rho=-0.9$

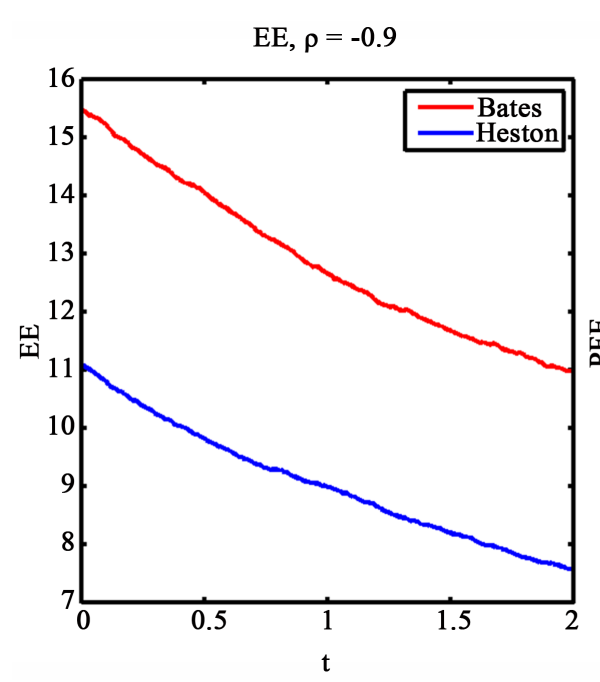

PFE, $\rho=0.9$

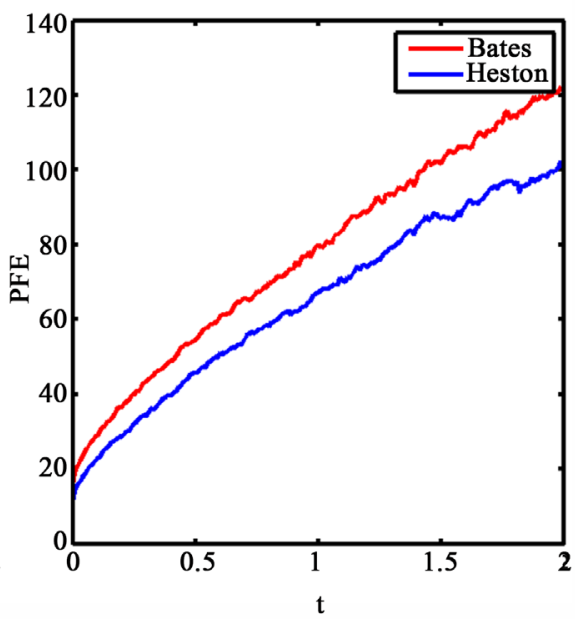

Figure 7. ATM option, $\rho=-0.9$.

explained by martingale theory proposed by Tang and Li [25]. However, when $\rho \neq 0$, the WWR EE is no longer lingering around the initial level. Figure 6 illustrates the case when $\rho=0.9$ and Figure 7 corresponds to the situation when $\rho=-0.9$. When correlation is counted, the EE either increase with time or decrease with time. That is exactly an outcome the WWR.

\subsubsection{Effect of Jump Parameters}

In this section, we consider the effect of the additional jump parameter entering the Bates model, namely the jump intensity $\lambda_{J}$ and the jump size volatility $\sigma_{J}$. In the left panel of Figure 8, EE profiles for different levels of $\sigma_{J}$ are plotted. We can see that increase of $\sigma_{J}$ causes the EE to increase significantly. The overall impact on CVA is listed in the right panel of Figure 8. As it shows that CVA increases from 1.49 to 2.06 when $\sigma_{J}$ increases from 0.1 to 1 , the impact of $\sigma_{J}$ is substantial. Study of $\lambda_{J}$ is shown in Figure 9. It can be noticed that both EE and CVA rise as jump intensity $\lambda_{J}$ increases. As we know, $\lambda_{J}$ de- 

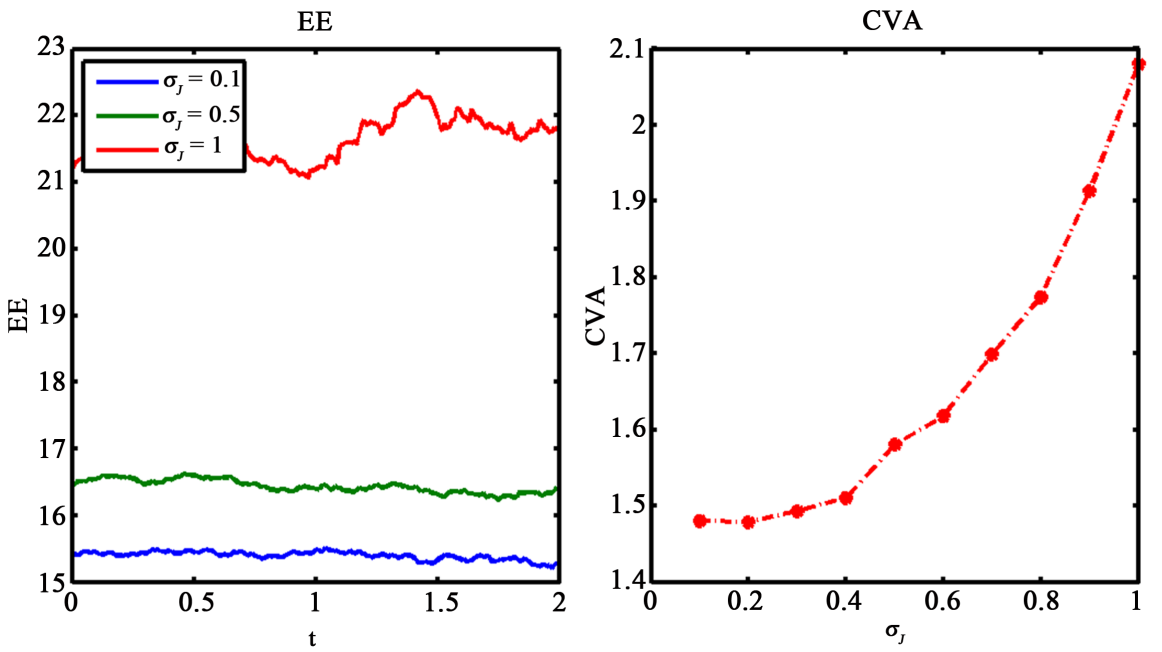

Figure 8. Impact of $\sigma_{J}$.
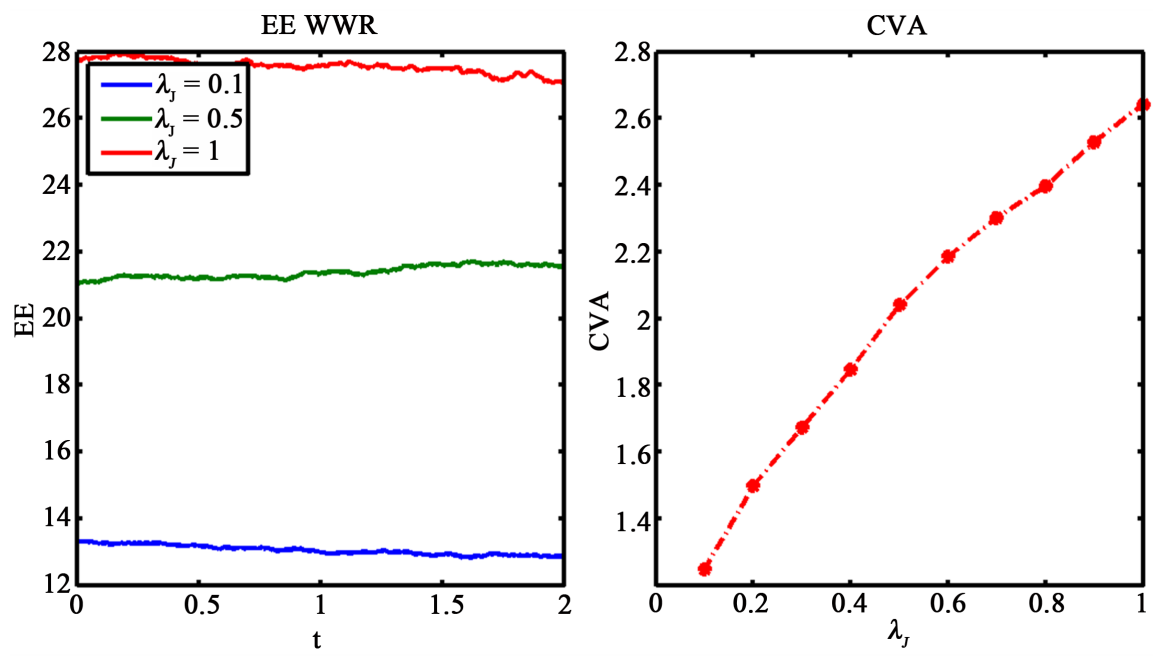

Figure 9. Impact of $\lambda_{J}$.

notes the jump frequency, CVA increase naturally with the increase of jump frequency.

All these results show that jump has an impact on exposure profiles as well as CVA. The introduction of jump-at-default can result in large jump WWR. Therefore, it is important for CVA and risk management purpose.

\section{Conclusions}

The expected exposure is an option on the market value of the position; if the position itself is an option, the evaluation is to price an option on the option; it will require the stochastic volatility to generate sufficient volatility of the option position and catch the risk properly. The developed Bates model with stochastic intensity of the jump to default in CIR++ process in this paper provides a natural and effective framework to generate the sufficient volatility and provide the jump to default feature which is essentially important for addressing the WWR. How to efficiently evaluate the expected exposure precisely and how to catch the 
WWR properly are big challenges to both financial industry and academia; in the past, many progresses have been made as pointed out in the introduction. In this paper, we develop and demonstrate an efficient PDE based Monte Carlo framework for pricing CVA and assessing exposure profile under Bates model with stochastic default intensity in $\mathrm{CIR}++$ process which combines the advantages of Monte Carlo simulation (such as path-wise pricing, and properly netting and collateral modeling) and efficiency of PDE pricing. The developed framework can be used for pricing both single trade and portfolios and it is a practically useful algorithm/framework in which its computation performance could be improved significantly by computing each individual path parallelly.

This work studies CVA in presence of WWR caused by the correlation between the underlying asset and the jump-to-default. The work can be further improved by including correlations between underlying asset, the jump to default, the option seller and the bank. This research focuses mainly on the equity asset and European option in our examples, however, the developed framework in this paper can be applied to price CVA and study WWR for other assets such as commodity, FX and credit etc. as well as other options such as Bermudan option, American option and barrier option etc.

\section{References}

[1] Basel Committee on Banking Supervision (2015) Review of the Credit Valuation Adjustment Risk Framework. http://www.bis.org/bcbs/publ/d325.pdf

[2] Basel Committee on Banking Supervision (2011) Press Release on Capital Treatment for Bilateral Counterparty Credit Risk Pointed out "During the Financial Crisis, however, Roughly Two-Thirds of Losses Attributed to Counterparty Credit Risk Were Due to CVA Losses and Only about One-Third Were Due to Actual Defaults". http://www.bis.org/press/p110601.htm

[3] Gregory, J. (2010) Counterparty Credit Risk: The New Challenge for Global Financial Markets. The Wiley Finance Series, New Haven, CT. Review, 37, 16-22.

[4] Feng, Q. and Oosterlee, C.W. (2012) Monte Carlo Calculation of Exposure Profiles and Greeks for Bermudan and Barrier Options under the Heston-Hull-White Model. https://arxiv.org/abs/1412.3623

[5] de Graaf, C.S., Feng, Q., Kandhai, D. and Oosterlee, C.W. (2014) Efficient Computation of Exposure Profiles for Counterparty Credit Risk. International Journal of Theoretical and Applied Finance, 17, 1-24.

https://doi.org/10.1142/S0219024914500241

[6] de Graaf, C.S., Kandhai, D. and Sloot, P.M. (2014) Efficient Estimation of Sensitivities for Counterparty Credit Risk with the Finite Difference Monte Carlo Method. https://papers.ssrn.com/sol3/papers.cfm?abstract_id=2521431

[7] Hull, J. and White, A. (2012) CVA and Wrong Way Risk. Review of Financial Studies, 3, 58-69. https://doi.org/10.2469/faj.v68.n5.6

[8] Pykhtin, M. and Rosen, D. (2010) Pricing Counterparty Risk at the Tradelevel and CVA Allocations. Journal of Credit Risk, 6, 3-38. https://doi.org/10.21314/JCR.2010.116

[9] Brigo, D. and Vrins, F. (2016) Disentangling Wrong-Way Risk: Pricing CVA via Change of Measures and Drift Adjustment. https://arxiv.org/abs/1611.02877

[10] Pykhtin, M. and Sokol, A. (2012) If a Dealer Defaulted, Would Anybody Notice. 
Global Derivatives 2012 Presentation.

https://www.compatibl.com/research/publications/systemic-wrong-way-risk/pykhti n\%20sokol\%20global\%20derivatives\%20barcelona\%202012.pdf

[11] Fabio, M. and Minqiang, L. (2015) Jumping with Default: Wrong-Way Risk Modelling for CVA. Journal of Credit Risk.

[12] Feng, L. and Linetsky, V. (2008) Pricing Options in Jump-Diffusion Models: An Extrapolation Approach. Operations Research, 56, 304-325. https://doi.org/10.1287/opre.1070.0419

[13] Pykhtin, M. and Zhu, S. (2007) A Guide to Modelling Counterparty Credit Risk. GARP Risk Review.

[14] Bocker, K. and Brunnbauer, M. (2014) Path-Consistent Wrong-Way Risk: Acopula-Based Model for Wrong Way Risk. Credit Risk Review.

[15] Brigo, D. and Pallavicini, A. (2008) Counterparty Risk and Contingent CDS Valuation under Correlation between Interest Rates and Default.

[16] Ruiz, I., Boca, P.D. and Pachon, R. (2015) Optimal Right- and Wrong-Way Risk from a Practitioner Standpoint. Financial Analyst's Journal, 71, 47-60. https://doi.org/10.2469/faj.v71.n2.1

[17] Bates, D.S. (1998) Jumps and Stochastic Volatility: Exchange Rate Processes Implicit in Deutsche Mark Options. Review of Financial Studies, 9, 69-107. https://papers.ssrn.com/sol3/papers.cfm?abstract_id=926067 https://doi.org/10.1093/rfs/9.1.69

[18] Almendral, A. and Oosterlee, C.W. (2005) Numerical Valuation of Options with Jumps in the Underlying. Applied Numerical Mathematics, 53, 1-18. https://doi.org/10.1016/j.apnum.2004.08.037

[19] Heston, S.L. (1993) A Closed-Form Solution for Options with Stochastic Volatility with Applications to Bond and Currency Options. The Review of Financial Studies, 6, 327-343. https://doi.org/10.1093/rfs/6.2.327

[20] Haentjens, T. and in't Hout, K.J. (2012) ADI Finite Difference Schemes for the Heston-Hull-White PDE. The Journal of Computational Finance, 16, 83-110. https://doi.org/10.21314/JCF.2012.244

[21] Hout, K.J.I.T. and Foulon, S. (2010) ADI Finite Difference Schemes for Option Pricing in the Heston Model with Correlation. International Journal of Numerical Analysis and Modeling, 7, 303-320.

[22] Santtu, S., Jari, T. and von Lina, S. (2013) Iterative Methods for Pricing American Options under the Bates Model. International Conference on Computational Science, 18, 1136-1144.

[23] Sjoberg, A. (2013) Adaptive Finite Differences to Price European Options under the Bates Model. http://www.diva-portal.org/smash/get/diva2:646051/FULLTEXT01.pdf

[24] Stamm, R., Gallagher, D. and Lichters, R. (2015) Modern Derivatives Pricing and Credit Exposure Analysis. Palgrave Macmillan, San Francisco.

[25] Tang, Y. and Li, B. (2007) Quantitative Analysis, Derivatives Modeling, and Trading Strategies: In the Presence of Counterparty Credit Risk for Fixed-Income Market. World Scientific Pub, New Haven. https://doi.org/10.1142/4228 
Submit or recommend next manuscript to SCIRP and we will provide best service for you:

Accepting pre-submission inquiries through Email, Facebook, LinkedIn, Twitter, etc. A wide selection of journals (inclusive of 9 subjects, more than 200 journals)

Providing 24-hour high-quality service

User-friendly online submission system

Fair and swift peer-review system

Efficient typesetting and proofreading procedure

Display of the result of downloads and visits, as well as the number of cited articles Maximum dissemination of your research work

Submit your manuscript at: http://papersubmission.scirp.org/

Or contact jmf@scirp.org 